\title{
KINETIC ASYMMETRY DURING RUNNING AT PREFERRED AND NON- PREFERRED SPEEDS
}

\author{
Laura-Anne M Furlong $^{1,2}$ * and Natalie L Egginton ${ }^{1}$
}

\begin{abstract}
Affiliations:
${ }^{1}$ School of Sport, Exercise and Health Sciences, Loughborough University, Loughborough, Leicestershire, LE11 3TU, United Kingdom;

${ }^{2}$ National Centre for Sport and Exercise Medicine - East Midlands, Loughborough, Leicestershire, LE11 3TU, United Kingdom
\end{abstract}

Address for correspondence: School of Sport, Exercise and Health Sciences, Loughborough University, Epinal Way, Loughborough, Leicestershire, LE11 3TU, United Kingdom

\section{Corresponding author:}

Dr. Laura-Anne M Furlong (address as above)

Tel: +44 (0) 1509223059

Fax: +44 (0) 1509226301

Email: L.A.M.Furlong@lboro.ac.uk

Running title: Kinetic asymmetry during running

Submitted type: Original investigation

Conflict of interest statement: There are no conflicts of interest associated with this research. The results of the present study do not constitute endorsement by ACSM. Results of this study are presented clearly, honestly, and without fabrication, falsification, or inappropriate data manipulation. 


\section{ABSTRACT}

PURPOSE: The aim of this study was to investigate the effect of altering preferred running speed by $\pm 20 \%$ on kinetic asymmetry.

METHODS: Three-dimensional motion analysis and force data were acquired from 15 healthy males (age: $27 \pm 4.6$ years, height: $1.81 \pm 0.09 \mathrm{~m}$, mass: $80.4 \pm 12.4 \mathrm{~kg}$ ) during their preferred running speed, and at $\pm 20 \%$ of this speed. 3 T magnetic resonance images were used to measure Achilles tendon cross-sectional area and moment arm, for use in calculation of tendon stress. Kinetic and tendon stress asymmetry were subsequently calculated in each condition using the symmetry index.

RESULTS: Across all joints and conditions, average asymmetry of peak moments was between $\pm 6 \%$ but higher individual values were observed; there was no effect of speed on magnitude of asymmetry. Ground contact times, vertical ground reaction forces and support and ankle moments (maximum absolute asymmetry: 9\%) were more symmetrical than hip and knee moments (up to 18\%). Individual joint contribution to support moment and positive work were similar in both limbs, and ankle and hip compensatory interactions were observed with alterations in running speed. Achilles tendon stress increased with increased running speed, with higher stress in the preferred limb; asymmetry in tendon stress was not related to asymmetry in vertical ground reaction forces.

CONCLUSION: Results show small effects of altering running speed on kinetic asymmetry, but responses are individual-specific with interactions occurring between joints to maintain overall movement symmetry. Further research is needed to 
understand the mechanical and neuromuscular mechanisms underpinning these compensations.

KEYWORDS: Injury, training program design, locomotion, loading, lower limb, stress, Achilles tendon 


\section{INTRODUCTION}

Running is one of the most popular recreational sports worldwide, with large-scale events across a range of distances attracting hundreds of thousands of participants. In 2016, over 133,000 people completed the New York, Paris and Chicago marathons combined, and over 40,000 people complete the $10 \mathrm{~km}$ Dublin MiniMarathon. To complete these events, individuals often join local running groups or train with friends. Group running forms a significant part of team sport and military fitness training, used as a time-efficient, team-building method of building cardiovascular fitness. However, it is highly improbable all will have the same leg length or preferred movement speed, meaning some individuals must run faster or slower than personally optimal to ensure the group stays together. Enforced running at non-preferred speeds may also occur while racing, where an athlete needs to move faster or slower than they would preferentially choose to, due to strategies employed by other competitors. How modifications in speed affect lower limb kinematics and kinetics, and in particular, whether both limbs are equally affected, is currently not well understood. How these modifications influence internal measures of loading such as tissue stress, which potentially leads to development of injury, is also unknown.

Between-limb asymmetry has traditionally been viewed negatively based on the work of Knapik et al., who reported asymmetry greater than $15 \%$ in isokinetic knee flexor strength and hip extensor flexibility was associated with increased injury risk in female collegiate athletes (1). However, recent work assessing asymmetries of gait, neuromuscular function and muscle-tendon mechanical properties in otherwise 
healthy populations suggest this value is conservative (2). While peak vertical ground reaction force (VGRF) differences are typically below 15\% (3-5), asymmetry of $23.3 \%$ for average vertical loading rate and $32.8 \%$ in peak mediolateral ground reaction force has been reported previously $(6,7)$. Furlong and Harrison (8) reported between-limb differences in plantarflexor force production capabilities of $14 \pm 8.6 \%$, and in rate of force production of up to $23 \pm 18.8 \%$. Similarly, Bohm et al. (9) reported average asymmetry of 3 to $31 \%$ in Achilles tendon morphology and mechanics in comparable healthy, recreationally active adults. Presence of asymmetry has been hypothesised to negatively influence injury risk due to potential unequal distribution of forces throughout the limb, but there is little longitudinal scientific evidence to support this $(4,5)$. This finding may in part be due to a lack of research interrogating the link between internal and external measures of asymmetrical musculoskeletal load. Furthermore, establishing if both limbs respond similarly to altered task demands provides fundamental insights into lower limb control and system organisation during dynamic tasks.

Understanding of asymmetry in gait is poor, both in establishing 'normal', healthy levels of asymmetry, and evaluating if asymmetrical external loading is indicative of asymmetrical internal loading. Most work investigating asymmetry utilises discrete point analysis of variables such as peak force, which excludes additional information related to the entire stance. It is unknown how subtle modifications of running speed, as may be observed in a group training setting, affect these metrics. The aim of this study was to investigate kinetic asymmetry during running at preferred and nonpreferred running speeds. Specific objectives were to investigate the effects of modification of running speed on between-limb kinetic asymmetry in magnitude and 
timing of peak vGRF, joint and support moments and Achilles tendon stress. It was hypothesised preferred and non-preferred limbs would exhibit similar responses at preferred and non-preferred running speeds, at whole-body, joint and muscle-tendon level, and asymmetry of external loading would be related to asymmetrical internal loading.

\section{METHODS}

\subsection{Participants}

Following institutional ethical approval, 15 able-bodied, healthy, male participants (mean \pm standard deviation; age: $27 \pm 4.6$ years, height: $1.81 \pm 0.09 \mathrm{~m}$, mass: $80.4 \pm$ $12.4 \mathrm{~kg}$ ) provided written informed consent to take part in this study. Participants were recreationally active, participating in at least 30 minutes of physical activity a day, 3 days per week; and were asked to refrain from unaccustomed strenuous exercise 24 hours proceeding testing. None had suffered lower limb injury in the preceding six months or had a history of lower limb surgery. Limb preference was determined as the limb preferentially used for two of single-leg balance, hopping and kicking tasks (10). Eleven participants preferred their right leg, and four the left. Post self-selected cardiovascular and stretching warm-up, participants completed at least three practice runs to establish preferred running speed (PRS), the speed they would complete a typical training run at, during over-ground running along a $12 \mathrm{~m}$ runway.

\subsection{Motion and force data acquisition}


A sixteen camera three-dimensional motion analysis system $(250 \mathrm{~Hz}, \mathrm{MX} 13$, Vicon Motion Systems Limited, Oxford, UK) synchronised to an AMTI force plate $(2000 \mathrm{~Hz}$, AMTI OR6, Advanced Mechanical Technology Inc., Massachusetts, USA) was used to acquire motion and force data. Twenty-two $14 \mathrm{~mm}$ reflective markers (B\&L Engineering, California, USA) defined the anatomical bony landmarks of both limbs, namely medial $1^{\text {st }}$ metatarsophalangeal head, lateral $5^{\text {th }}$ metatarsophalangeal head, superior distal hallux, calcaneus, medial and lateral malleolus, medial and lateral knee joint centre, greater trochanter, and the anterior and posterior iliac crests. Participants wore their own running shoes, and starting position was adjusted to permit force plate contact during the trial using the participants natural stride pattern without conscious adjustment. Three trials where the preferred and non-preferred limb contacted the force plate at the three different test speeds (i.e. 9 trials for each limb, 18 in total) were completed in a block randomised order to minimise order effects. Trials were completed at PRS-20\%, PRS and PRS+20\%, with the force plate zeroed between trials to minimise drift. Running speeds were measured using Smart Speed timing gates (Fusion Sport, Queensland, Australia) located $0.75 \mathrm{~m}$ either side of the centre of the force plate, at the height of the participant's estimated centre of mass. Trials were performed to within $\pm 5 \%$ of the pre-determined target speed.

\subsection{Magnetic resonance imaging (MRI)}

Axial spin-echo T1 weighted images (TR/TE: 840/7.7, FOV: $45 \times 45 \mathrm{~cm}$, matrix: 512x512, slice thickness: $5 \mathrm{~mm}$, spacing $0 \mathrm{~mm}$ ) were obtained using a 3 Tesla scanner (Discovery MR750W, GE Healthcare, Fairfield, CT., USA). Both limbs were scanned simultaneously from the iliac crest to the dorsum of the foot. Small variations in TR/TE settings were necessary for some individuals to optimise image 
quality. A standardised supine position was used for all participants with the feet, knees and hips supported by solid foam cushions to achieve neutral ankle and knee angles and prevent hip external rotation. Full lower limb data were acquired in four scanning blocks. Standardisation of where the tendon cross-sectional area (CSA) measurements were to be obtained was achieved by placing a single fish oil capsule $6 \mathrm{~cm}$ proximal to the calcaneal insertion of the Achilles tendon.

\subsection{Data processing}

Motion analysis marker data were reconstructed and labelled using Vicon Nexus 1.8.5 (Vicon Motion Systems Limited, Oxford, UK), with any missing marker data of up to 10 frames filled using a quintic spline. All marker data were filtered using a fourth order, reverse pass, low pass Butterworth filter using a cut-off frequency of 12 $\mathrm{Hz}$, determined using residual analysis (11); force data were not filtered. Forces, moments and powers at the ankle, knee and hip during stance, defined as when the vGRF was greater than a $10 \mathrm{~N}$ threshold, were calculated using an inverse dynamics approach in BodyBuilder (Vicon Motion Systems Limited, Oxford, UK) and normalised to $100 \%$ of stance. Hip, knee and ankle extensor moments were expressed as positive, and the support moment was calculated as the algebraic sum of these moments (12). Timing of the peak vGRF, support, ankle, knee and hip moments was determined as a percentage of total ground contact time.

Between-limb differences in peak values were calculated using the symmetry index, where the difference between the preferred and non-preferred limb was expressed as a percentage of the average of the preferred and non-preferred limbs (13). Use of this calculation may artificially inflate calculated asymmetry of small values, but 
provides an easily interpretable value for the practitioner or clinician to understand. The absolute value of this index was also calculated, due to positive and negative indices potentially cancelling each other out during group averaging. The root mean square difference (RMSD) between the mean ensemble curves of vertical vGRF, support and joint moments for the preferred and non-preferred limbs in each condition was calculated to quantify the difference between the two limbs across the entire stance phase. This value was then expressed as a percentage of the average of the preferred and non-preferred peak values for that particular moment to provide a normalised value for each individual.

Contributions from individual joints during stance were assessed both in resisting collapse of the limb (support moment) and in contributions to total average positive power (as an indication of contribution to propulsion). The instance of peak support moment was identified and relative proportion of this value contributed by each joint then calculated. Contribution to total average positive power was calculated using a similar method to Farris and Sawicki (14) and Schache et al. (15). Briefly, joint powers were integrated with respect to time using the trapezium method to calculate joint work, and all periods of positive work at an individual joint summed to give total joint positive work. These values were then divided by stance time to calculate average positive mechanical joint power. Average positive joint powers were summed to give total average positive power output, with each joint's contribution subsequently expressed as a percentage of this value.

Peak Achilles tendon stress was calculated by dividing calculated tendon force by CSA. Estimated peak tendon force at the region of interest $(6 \mathrm{~cm}$ proximal to the 
calcaneus) was calculated by dividing the peak plantarflexor moment by the Achilles tendon moment arm, assuming negligible co-contraction of the flexors and that $100 \%$ of this force was transmitted through the tendon at the point of interest. Moment arms were calculated from the MR images as the perpendicular distance between the ankle joint centre (midpoint of the medial and lateral malleoli) and the line of action of the Achilles tendon. There are known differences in moment arm length with change in joint angle, but it was assumed these changes would be constant across all individuals. Tendon CSA was quantified by outlining the boundaries of the tendon in a continuous trace method in ImageJ 1.50i (National Institutes of Health, Maryland, USA), following software calibration to a known $10 \mathrm{~mm}$ distance. The average of three trials for each image was used for subsequent calculations. Maximum coefficient of variation of these data was $1.2 \%$.

\subsection{Statistical analysis}

Descriptive statistics (mean and standard deviation) were calculated in Microsoft Excel 2016 (Microsoft Corp., Redmond, WA., USA). All further statistical analyses were completed in SPSS Statistics 22 (IBM, Armonk, NY, USA). The assumptions of normality, homogeneity of variance and sphericity were assessed using ShapiroWilk's test, Levene's test and Mauchly's test of sphericity. Paired sample t-tests and repeated measures analysis of variance were used to determine if between-limb or between-condition differences were statistically significant $(\alpha \leq 0.05)$. Where data did not satisfy the assumption of sphericity, Greenhouse-Geisser corrections were used. Null hypotheses tested were: 1) magnitudes and timings of peak vGRF, support and joint moments of the preferred and non-preferred limbs were not affected by running speed, and 2) magnitudes and timings of peak vGRF, support and joint moments at 
a given running speed were not affected by limb preference. Pearson's correlation coefficient was used to test the null hypothesis that there was no relationship between Achilles tendon stress, and stress asymmetry with vGRF and vGRF asymmetry.

Statistical parameter mapping (SPM) analysis of variance with repeated measures was used to statistically evaluate the effect of running speed on between-limb differences in support and joint moments across the entire stance phase. This analysis utilised a similar alpha level as discrete point statistical analyses $(\alpha \leq 0.05)$. All SPM analyses were implemented using the open-source spm1d code (v.M0.1, www.spm1d.org) in Matlab (R2015a, The Mathworks Inc, Natick, MA., USA). The scalar output statistic, $\operatorname{SPM}\{F\}$, was calculated separately at each individual time node and referred to as a Statistical Parametric Map. A critical threshold, where only $5 \%$ of smooth random curves were expected to traverse, was calculated based upon estimates of trajectory smoothness via temporal gradients (16) and Random Field Theory expectations regarding the field-wide maximum (17). The null hypothesis tested was between-limb differences in vGRF, support and individual joint moments at each instant in time across the stance were not affected by alteration in running speed. The null hypothesis was rejected if the $\operatorname{SPM}\{F\}$ trajectory crossed the critical threshold at any time node in an analysis.

\section{RESULTS}

Average running speeds across all subjects were $2.5 \pm 0.5,3.1 \pm 0.6$ and $3.7 \pm 0.7 \mathrm{~m} . \mathrm{s}^{-1}$ for the PRS-20\%, PRS and PRS $+20 \%$ conditions. With this increase 
in running speed, there was a statistically significant $(p<0.001)$ decrease in average ground contact time. Ground contact times for the preferred and non-preferred limbs were 322 and 318 ms, 270 and 268 ms, and 238 and 245 ms during the PRS-20\%, PRS and PRS $+20 \%$ conditions. Between-limb differences were not statistically significant with the exception of the PRS $+20 \%$ condition $(p<0.05)$.

Statistically significant increases in vGRF, support and joint moments with increased running speed were observed in both limbs $(p<0.01)$. Mean values (normalised to body mass) during each condition for peak ankle, knee and hip extensor moment, and support moment are presented in Figure 1, with all original data presented to indicate the inconsistency in directionality of differences; how calculated asymmetry changed for each variable for each participant is presented in Table 1. Group average asymmetry ranged from $-6 \%$ to $+6 \%$ across all speeds, forces and moments. Although several between-limb differences were statistically significant (peak vGRF at PRS-20\%, support moment at PRS-20\% and PRS+20\%, knee at PRS+20\%, and ankle at PRS-20\% and PRS, $\mathrm{p}<0.05)$; this is more reflective of less variable data than of clinically meaningful difference. Maximum average absolute differences were $3 \pm 6 \%$ (vGRF, PRS+20\%), $9 \pm 6 \%$ (support moment, PRS+20\%), $18 \pm 18 \%$ (hip, PRS+20\%), $13 \pm 8 \%$ (knee, PRS-20\%) and $8 \pm 6 \%$ (ankle, PRS+20\%).

Speed had a significant effect on timing of the peak support and joint moments $(p<$ 0.01) with the exception of the non-preferred hip and knee, but between-limb differences were not statistically significant with the exception of the peak ankle moment at PRS $(p<0.05)$. Peak support moment occurred on average at $36 \%$ (both limbs), 38\% (both limbs), and 38\% (preferred limb) and 39\% (non-preferred limb) of 
stance during each condition. Small average between-limb absolute differences in timing of $2 \%$ were observed across all peak values (maximum absolute differences: 6,7 and $5 \%$ in each condition). There was no effect of speed on the contributions of the ankle or knee to the support moment, but there was a statistically significant effect on the hip ( $p<0.05)$; changes in contribution to positive power from the hip and ankle with change in speed were statistically significant $(p<0.05)$ but changes at the knee were not (Figure 2). Between-limb differences in contribution to support from the knee during PRS-20\% and to positive power at the ankle during PRS-20\% were also statistically significant $(p<0.05)$, but all others were not. Smaller ankle contributions were associated with higher hip contributions; a trend for smaller contributions in the preferred limb and greater contributions from the preferred hip, compared with those of the non-preferred limb, was observed at all speeds.

Across the entire stance phase, average normalised RMSD between preferred and non-preferred mean ensemble curves were low with average differences of below $13 \%$, but individual maximum values tended to be high with maximum differences of up to $27 \%$ of the peak moment observed at the knee (PRS+20\%, Table 2). Values were lower for the vGRF and ankle moments than the knee and hip moments. There was no statistical effect of speed on asymmetry observed between any force or moment curves.

Achilles tendon moment arm of the preferred limb was $49.1 \pm 3.7 \mathrm{~mm}$, and $48.1 \pm 3.6$ $\mathrm{mm}$ in the non-preferred, with tendon CSA of $80.4 \pm 1.01 \mathrm{~mm}^{2}$ and $83.61 \pm 0.93 \mathrm{~mm}^{2}$ respectively. Achilles tendon stresses were $49.5 \pm 9.2,54.5 \pm 9.6,58.4 \pm 14.3 \mathrm{MPa}$ for the preferred limb at each speed, and $46.5 \pm 10.1,51.5 \pm 12.4$ and $56.2 \pm 13.9$ for the 
non-preferred limb. Average asymmetry was $7 \pm 23.9 \%, 7 \pm 18.9 \%$, and $4 \pm 25.3 \%$ for PRS-20\%, PRS, and PRS+20\%; large average absolute differences were observed of $20 \pm 14.8 \%, 16 \pm 11.1 \%$, and $19 \pm 16.7 \%$. Effect of speed on tendon stress was statistically significant $(p<0.001)$ but between-limb differences were not. Correlations between Achilles tendon stress and peak vGRF were strong and statistically significant in the preferred limb during PRS-20\% $(r=0.553)$ and PRS $(r=$ 0.709), and non-preferred limb at PRS-20\% ( $r=0.739)$, but correlations between asymmetry of each variable were low and non-significant $(r<0.38)$.

\section{DISCUSSION}

This study is one of the first to present normative kinetic asymmetry values for running at a preferred movement speed and with subtle alterations to this speed, as is likely to occur when training as part of a group or during a race event. Peak moments increased and ground contact time decreased as expected with an increase in running speed. Although some statistically significant between-limb differences in magnitude and timing of peak moments and across the entire stance phase were observed, these differences were small. Several large individual-specific responses were found. Asymmetrical internal loading was not related to asymmetrical external measures of load.

Reported running speeds, contact times, and moment values are similar to those previously reported in the literature $(14,18)$. The average low level of asymmetry observed for both peak moments and across the entire stance (all $\leq 12 \%$ ) was comparable to that previously observed in the literature $(4,19)$ with no group 
average greater than $\pm 6 \%$ in the variables of interest. Particularly at joint level, large maximum absolute differences were observed. Overall, lower asymmetry was observed in gross outcome measures such as ground contact time (average absolute asymmetry of 5\%, 3\% and $5 \%$ in each condition), vGRF (average absolute asymmetry of $3 \%$ in each condition) and support moment (average absolute asymmetry of $8 \%, 6 \%$ and $8 \%$ in each condition) than at the individual joints. The magnitude of between-limb differences in peak joint kinetics was dependent on the particular moment of interest. The support moment was proposed by Winter (12) as a measure of the resistance of the limb to collapse, and it is reasonable to expect both limbs would demonstrate similar capacity to resist collapse. If asymmetry is high at one joint, some form of compensation must occur at other joints either within or between limbs to result in overall limb symmetry (20). Figure 2 graphically illustrates a form of compensation between the ankle and hip during positive power production, where the knee contribution remains constant but high ankle contribution is accompanied by lower hip, and vice versa. It is reasonable to assume similar interactions also occur for the joint moments. These results support the hypothesis suggested by Furlong and Harrison (8) to explain how asymmetry in plantarflexor force production capabilities may potentially by compensated for by more proximal joints. This study highlights the complexity of the human neuromusculoskeletal system and the need for an improved understanding of the organisation of the system, as well as highlighting the importance of assessing the asymmetry of the variable of interest in the task of interest. Interestingly, higher asymmetry was observed at the knee and hip joints, potentially due to the larger muscle mass acting around these joints and hence increased numbers of alternative muscle activation 
strategies to generate the same movement, but further research is needed in this area to fully understand these mechanisms.

Table 1 clearly illustrates that despite overall group mean similarity, individual responses were much more varied. Calculated symmetry index varied within an individual, dependent on the variable and condition of interest (Table 1), which highlights the difficulty in establishing one single cut-off value for a 'normal' level of asymmetry. Altering movement speed from preferred to non-preferred speeds will influence muscle activation and co-ordination, and force muscle to work at different parts of the force-velocity curve. The magnitudes of these changes will vary between individuals, and so it is not surprising as to why inconsistencies in the directionality of changes were observed both within and between individuals. Further study utilising measures of neuromuscular function and co-ordination during these tasks are justified to identify and further understand what modulates observed asymmetry (21).

Regardless of speed, at the instant of peak support moment there was little change in the contribution of the individual joints to the support moment. The timing of this peak was also consistent between conditions and limbs. The small change in velocity $\left(2.5\right.$ to $3.7{\mathrm{~m} . \mathrm{s}^{-1}}^{-1}$ may simply not have been sufficient to induce changes in the contribution required to resisting collapse by individual joints. However, with increases in speed, the ankle and hip, significantly changed their contribution to positive power (ankle decreased by $4 \%$ with increased speed, hip increased by 5$6 \%$ ). Magnitude of individual contributions observed here are comparable to those observed by Schache et al. $(15,22)$. The ankle is an important joint during 
acceleration (23) and with increased hopping frequency (24), and the hip muscles are the largest and most powerful in the body. It is hence not surprising to see changes in the role of these muscles with changes in speed. It must be noted the peak support moment is one instant in time; hence this analysis may not be indicative of what happens throughout the entire stance phase or indeed what may happen over the course of a 30-60 minute run. Over the course of a longer run, it is possible distributions may alter as different muscle groups fatigue, which could potentially be injurious for untrained structures.

Similar increases in average peak Achilles tendon stress were observed between conditions, with stress increasing by $10 \%$ as participants increased speed from PRS$20 \%$ to PRS, and by a further $7-10 \%$ as speed increased to PRS+20\%. Stress values observed here (49-56 MPa) are comparable to those reported by Lyght et al. (18) using musculoskeletal modelling during overground running at comparable speeds, and highlight the very high loads the Achilles tendon undergoes during dynamic tasks. CSA was measured at $6 \mathrm{~cm}$ proximal to the calcaneus as this has previously been identified as a key anatomical location for development of midportion Achilles tendinopathy. Current technology does not allow for simple, accurate, instantaneous measurement of tendon CSA during dynamic tasks such as running, but it is known that as the tendon is placed under load, CSA decreases as it elongates. Values here may hence potentially underestimate actual stress, a situation physiologically possible as previous work has reported peak stress of over $81 \mathrm{MPa}$ in the Achilles during longer duration isometric contractions (25). Ker et al. (26) reported a rupture stress of $100 \mathrm{MPa}$ for tendon. The safety factor of tendon refers to the ratio of the rupture stress of the tissue to observed stress during testing; 
participants in this study hence had a safety factor of just under 2 which is much lower than that of other tendons in the body during activity, and provides experimental evidence for why the Achilles tendon is so commonly injured.

While average peak stress asymmetry was low (maximum of $7 \%$ across the three conditions), the absolute average was a minimum of $16 \%$, with a wide range of values observed. The lack of relationship between VGRF and tendon stress asymmetry highlights an important potential explanation for the lack of scientific evidence that asymmetry is an important metric to predict injury risk. External load certainly has some influence on internal loading, as evidenced by the high correlations within a limb between VGRF and peak stress, but internal musculoskeletal loading is the result of multiple factors such as the structure (e.g. CSA, volume, tissue type) and mechanical properties (e.g. tissue stiffness, strain), both of whom are known to vary significantly from person to person (27). It is hence very difficult to accurately quantify the potential load on an individual structure such as the Achilles tendon based on solely external measures such as VGRF, and accurately predict its likelihood of injury. This highlights the need for further research investigating in vivo muscle mechanics during dynamic activities such as running to fully understand why tissues become injured during these activities, and importantly, what potential metrics may be helpful to identify someone likely to develop an injury.

Results show small differences between kinetics of the preferred and non-preferred limbs of healthy, active, young adult males during running at preferred and nonpreferred running speeds. However, individual-specific responses were observed. 
Joints of both limbs act equally to resist collapse and contribute to propulsion, and there appears to be a form of compensation between the ankle and hip when contributing to positive power across the stance phase. Peak Achilles stress asymmetry is not related to asymmetry in the vGRF, and highlights the importance understanding fundamental muscle mechanics and structure in identifying potential risk factors of injury. The results of this study cannot be extrapolated to understand the responses of previously injured individuals or female athletes to changes in running speed, or to provide insight into what happens over the course of a training run of 30 or 60 minutes duration. Future work is needed to establish if asymmetry is related to injury risk, investigate how kinetic asymmetry changes during and following longer-duration runs at non-optimal speeds, and further investigate the neuromechanical compensatory mechanisms used by an individual during these tasks. 


\section{REFERENCES}

1. Knapik J, Bauman C, Jones B, Harris J, Vaughan L. Preseason strength and flexibility imbalances associated with athletic injuries in female collegiate athletes. American Journal of Sports Medicine. 1991;19:76-81.

2. Sadeghi H, Allard P, Prince F, Labelle $H$. Symmetry and limb dominance in able-bodied gait: a review. Gait and Posture. 2000;12:34-45.

3. Brughelli M, Cronin J, Mendiguchia J, Kinsella D, Nosaka K. Contralateral leg deficits in kinetic and kinematic variables during running in Australian Rules football players with previous hamstring injuries. Journal of Strength and Conditioning Research. 2010;24(9):2539-44.

4. Bredeweg SW, Buist I, Kluitenberg B. Differences in kinetic asymmetry between injured and noninjured novice runners: A prospective cohort study. Gait and Posture. 2013;38(4):847-52.

5. Zifchock R, Davis I, Higginson J, McCaw S, Royer T. Side-to-side differences in overuse running injury susceptibility: a retrospective study. Human Movement Science. 2008;27:888-902.

6. Herzog W, Nigg B, Read L, Olsson E. Asymmetries in ground reaction force patterns in normal human gait. Medicine and Science in Sports and Exercise. 1989;21(1):110-4.

7. Zifchock R, Davis I, Hamill J. Kinetic asymmetry in female runners with and without retrospective tibial stress fractures. Journal of Biomechanics. 2006;39:27927.

8. Furlong L-AM, Harrison AJ. Differences in plantarflexor function during a stretch-shortening cycle task due to limb preference. Laterality: Asymmetries of Body, Brain and Cognition. 2015;20(2):128-40.

9. Bohm S, Mersmann F, Marzilger R, Schroll A, Arampatzis A. Asymmetry of Achilles tendon mechanical and morphological properties between both legs. Scandinavian Journal of Medicine and Science in Sports. 2015;25(1):124-32.

10. Chapman J, Chapman L, Allen J. The measurement of foot preference. Neuropsychologia. 1987;25(3):579-84.

11. Winter DA. Biomechanics and Motor Control of Human Movement. 3rd ed. New York: John Wiley and Sons Inc.; 2005. p. 49-50.

12. Winter DA. Overall principle of lower limb support during stance phase of gait. Journal of Biomechanics. 1980;13(11):923-7.

13. Robinson R, Herzog W, Nigg B. Use of force platform variables to quantify the effects of chiropractic manipulation on gait symmetry. Journal of Manipulative and Physiological Therapeutics. 1987;10:172-6.

14. Farris D, Sawicki G. The mechanics and energetics of human walking and running: a joint level perspective. Journal of the Royal Society: Interface. 2012;9:110-8.

15. Schache A, Dorn T, Williams G, Brown N, Pandy M. Lower-limb muscular strategies for increasing running speed. Journal of Orthopaedic and Sports Physical Therapy. 2014;44(10):813-24.

16. Friston K, Ashburner J, Kiebel S, Nichols T, Penny W. Statistical Parameter Mapping: The Analysis of Functional Brain Images. London, UK: Elsevier; 2007.

17. Adler R, Taylor J. Random Fields and Geometry. New York, USA: SpringerVerlag; 2007 
18. Lyght M, Nockerts M, Kernozek T, Ragan R. Effect of foot strike and step frequency on Achilles tendon stress during running. Journal of Applied Biomechanics. 2016;32(4):365-72.

19. Robinson R, Herzog W, Nigg B. Use of force platform variables to quantify he effects of chiropractic manipulation on gait asymmetry. Journal of Manipulative and Phsiological Therapeutics. 1987;10(4):172-6.

20. Exell T, Irwin G, Gittoes M, Kerwin D. Strength and performance asymmetry during maximal velocity sprint running. Scandinavian Journal of Medicine and Science in Sports. 2017;27(11):1273-82.

21. Carpes F, Mota C, Faria I. On the bilateral asymmetry during running and cycling - A review considering leg preference. Physical Therapy in Sport. 2010;11:136-42.

22. Schache A, Blanch P, Dorn T, Brown N, Rosemond D, Pandy M. Effect of running speed on lower $\operatorname{limb}$ joint kinetics. Medicine and Science in Sports and Exercise. 2011;43(7):1260-71.

23. Johnson MD, Buckley JG. Muscle power patterns in the mid-acceleration phase of sprinting. Journal of Sports Sciences. 2001;19(4):263-72.

24. Hobara H, Inoue K, Omuro K, Muraoka T, Kanosue K. Determinant of leg stiffness during hopping is frequency-dependent. European Journal of Applied Physiology. 2011;111:2195-201.

25. Waugh C, Blazevich A, Fath F, Korff T. Age-related changes in mechanical properties of the Achilles tendon. Journal of Anatomy. 2012;220:144-55.

26. Ker R, Wang X, Pike A. Fatigue quality of mammalian tendons. Journal of Experimental Biology. 2000;203:1317-27.

27. Lichtwark GA, Wilson AM. In vivo mechanical properties of the human Achilles tendon during one-legged hopping. Journal of Experimental Biology. 2005;208:4715-25. 

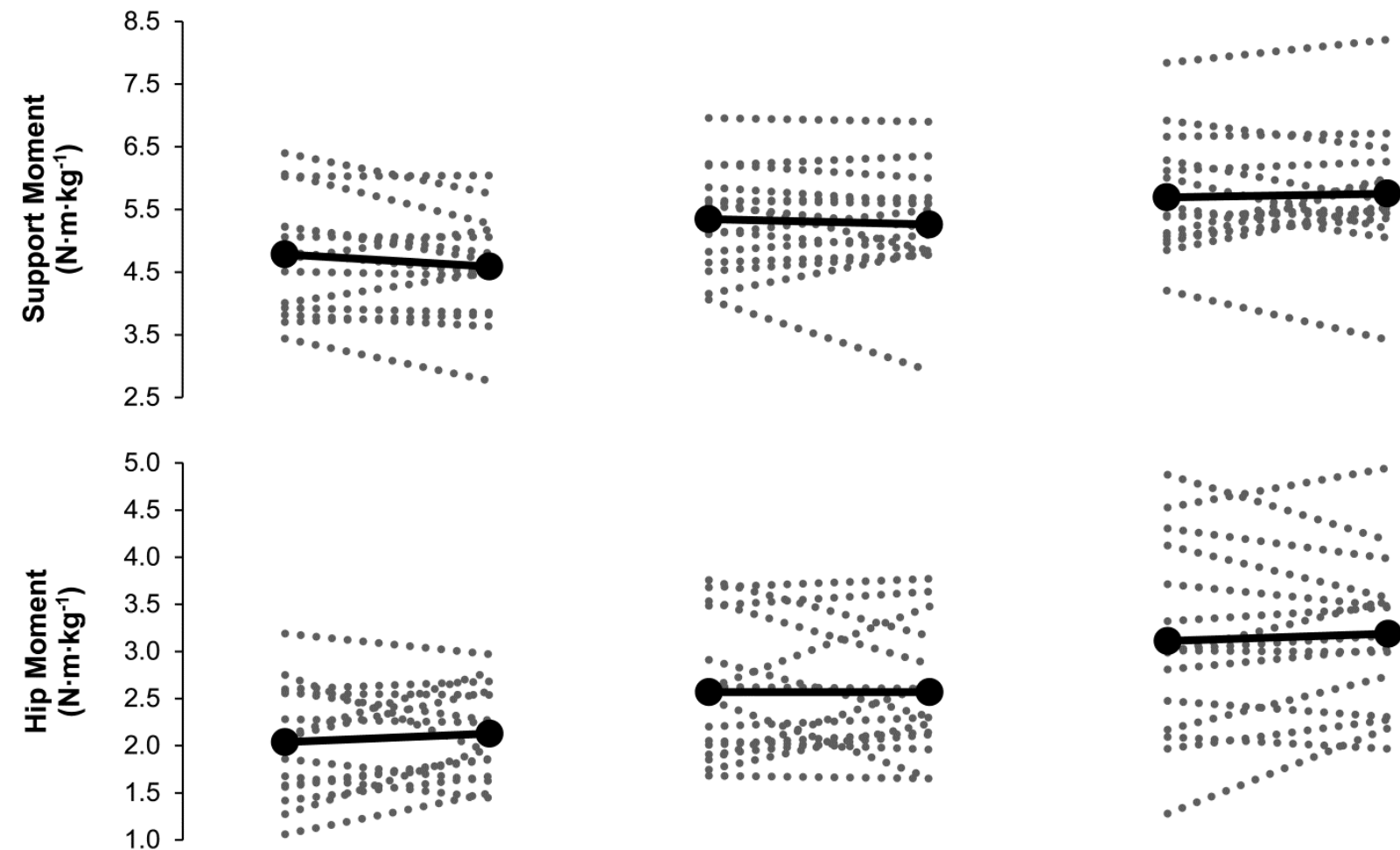

$\therefore: 800000 \div$

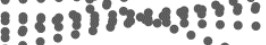
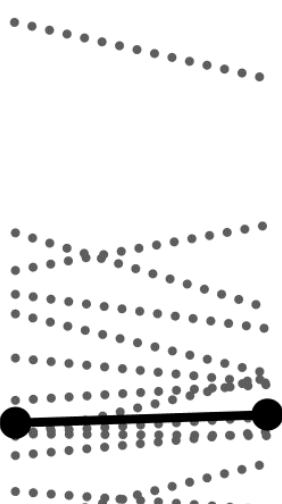

$8: \div 0008 \div: 88$

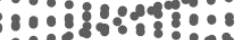
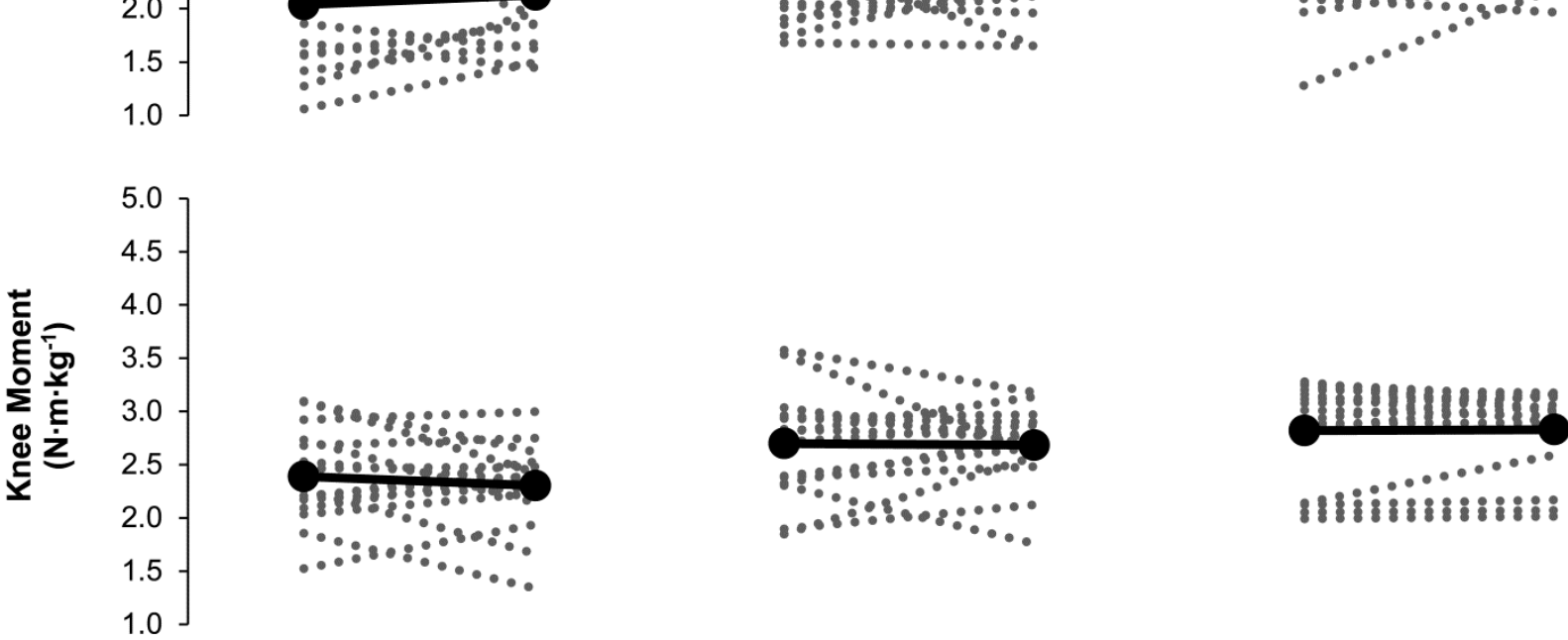

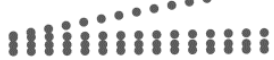

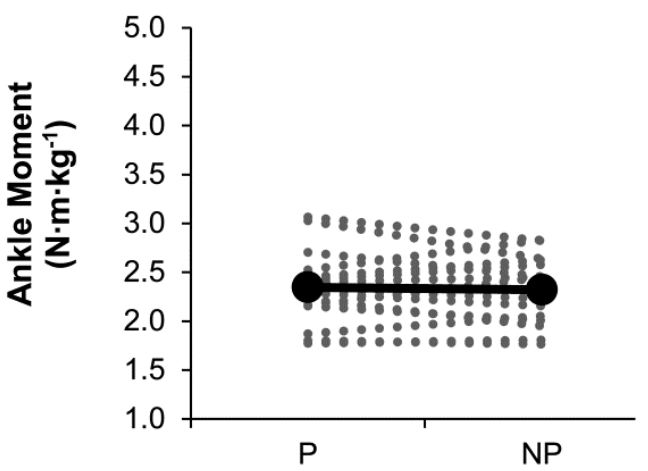

8ะ::::::::::::88
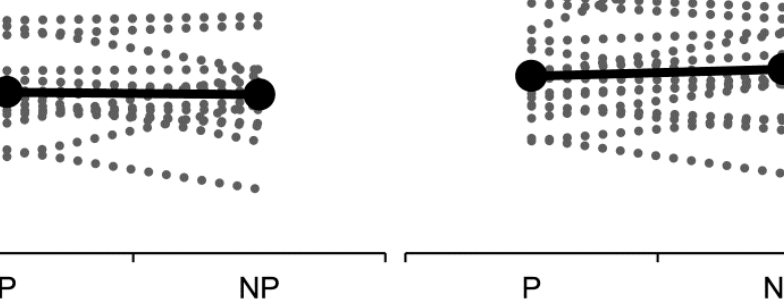

$\mathrm{P}$

Limb Preference 
Figure 1. Normalised support, hip, knee and ankle moments for preferred and nonpreferred limbs, during preferred running speed $-20 \%$, preferred running speed, and preferred running speed $+20 \%$. Solid black dots indicate group average values, and gray dotted lines indicate paired individual values. 


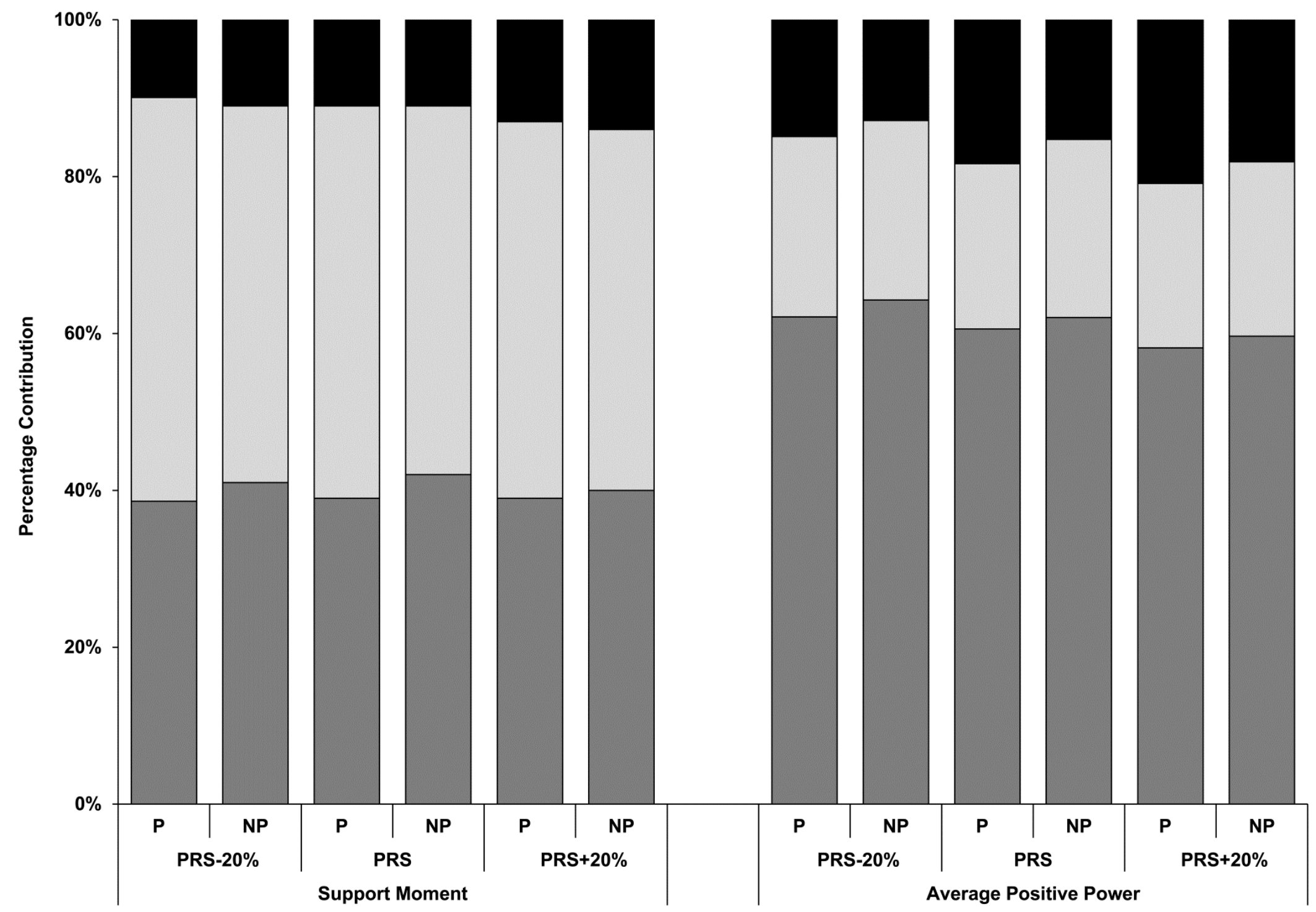

Figure 2. Percentage of peak support moment and average positive power contributed by the ankle (dark gray), knee (light gray), and hip (black) joints. 
Table 1. Symmetry indices for all participants in each condition, and calculated group average and standard deviation (units: percent)

\begin{tabular}{|c|c|c|c|c|c|c|c|c|c|c|c|c|c|c|c|c|c|c|c|c|c|}
\hline \multirow{2}{*}{$\begin{array}{l}\text { Participant } \\
\text { number }\end{array}$} & \multicolumn{3}{|c|}{ Ground contact time } & \multicolumn{3}{|c|}{$\begin{array}{l}\text { Peak vertical ground } \\
\text { reaction force }\end{array}$} & \multicolumn{3}{|c|}{ Peak support moment } & \multicolumn{3}{|c|}{ Peak hip moment } & \multicolumn{3}{|c|}{ Peak knee moment } & \multicolumn{3}{|c|}{ Peak ankle moment } & \multicolumn{3}{|c|}{$\begin{array}{l}\text { Peak Achilles tendon } \\
\text { stress }\end{array}$} \\
\hline & PRS-20 & PRS & PRS+20 & PRS-20 & PRS & PRS +20 & PRS-20 & PRS & PRS +20 & PRS-20 & PRS & PRS+20 & PRS-20 & PRS & PRS +20 & PRS-20 & PRS & PRS+20 & PRS-20 & PRS & PRS +20 \\
\hline P1 & -4 & -3 & -3 & 8 & 0 & -2 & 9 & 1 & -5 & 4 & 15 & 10 & 19 & 15 & 1 & -8 & -16 & -6 & -13 & 9 & 0 \\
\hline P2 & -8 & -2 & -12 & -2 & -7 & -3 & 4 & -7 & -5 & 7 & 21 & -23 & 21 & -31 & -6 & -7 & 2 & -13 & 56 & 46 & 39 \\
\hline P3 & 5 & 4 & 3 & -4 & 0 & 2 & -11 & -7 & -7 & -20 & -8 & -5 & -15 & -2 & -11 & -11 & -10 & -4 & 16 & 23 & 32 \\
\hline P4 & -2 & -4 & -6 & 7 & 1 & 6 & -10 & -1 & -11 & -10 & 0 & -9 & 1 & -10 & -36 & -9 & 0 & -1 & -4 & 11 & 2 \\
\hline P5 & 12 & -1 & -7 & 5 & 14 & -6 & 1 & 11 & 6 & 8 & 24 & 70 & 22 & 17 & 0 & -10 & -6 & 1 & -2 & 11 & -1 \\
\hline P6 & -2 & 4 & 1 & 5 & 3 & 7 & -6 & -2 & -3 & 20 & 13 & -11 & 2 & 6 & -8 & -12 & -9 & 0 & 20 & 16 & 9 \\
\hline P7 & -2 & 2 & 2 & -7 & -2 & 7 & -3 & 0 & -13 & -15 & 2 & -28 & 7 & 8 & 3 & -8 & -8 & -11 & 9 & 4 & 6 \\
\hline P8 & 11 & 5 & -6 & 6 & 0 & 1 & -3 & -1 & -3 & 11 & 30 & 18 & -1 & 8 & -10 & 1 & -9 & -6 & -15 & -3 & -23 \\
\hline P9 & -1 & 2 & -4 & 2 & 1 & -9 & 3 & 6 & -3 & -19 & 8 & 18 & 11 & 4 & -10 & -3 & -2 & -6 & -2 & -13 & -7 \\
\hline P10 & -3 & -4 & -8 & 6 & 6 & 1 & -12 & -15 & -12 & 18 & 8 & 39 & -16 & -25 & -7 & -7 & 0 & -11 & 22 & 20 & 33 \\
\hline P11 & -3 & -4 & -9 & 1 & -8 & -18 & -6 & 7 & 20 & 3 & -41 & 4 & 13 & 20 & 5 & -3 & 2 & 21 & -37 & -27 & -58 \\
\hline P12 & 5 & 0 & -4 & 2 & 7 & -3 & 6 & 2 & -4 & 7 & -8 & 15 & 18 & -1 & -1 & -5 & 2 & -14 & 39 & 10 & 30 \\
\hline P14 & 8 & 3 & -4 & 6 & 4 & -3 & -11 & -4 & 4 & 44 & -4 & 2 & 5 & 8 & 4 & -12 & -9 & 3 & 19 & 20 & 11 \\
\hline P15 & -8 & 3 & 4 & 1 & 2 & 1 & -15 & -21 & -21 & 29 & 0 & -9 & -23 & -15 & 0 & -4 & -16 & -15 & 16 & -3 & 13 \\
\hline Average & 1 & 0 & -3 & 3 & 2 & -1 & -5 & -2 & -4 & 4 & 4 & 6 & 3 & 0 & -6 & -6 & -5 & -4 & 7 & 7 & 4 \\
\hline $\begin{array}{l}\text { Standard } \\
\text { deviation }\end{array}$ & 6.4 & 3.2 & 4.8 & 4.8 & 5.4 & 7.0 & 7.9 & 8.2 & 9.5 & 19.7 & 17.1 & 24.8 & 15.2 & 14.8 & 10.6 & 4.2 & 6.6 & 9.6 & 23.9 & 18.2 & 25.3 \\
\hline
\end{tabular}


Table 2. Normalised root mean square differences between mean ensemble curves of vertical ground reaction force and extensor moment data for preferred and non-preferred limbs, expressed as a percentage of the average of preferred and non-preferred maximum values. Data presented is average \pm standard deviation with maximum difference in brackets.

\begin{tabular}{|c|c|c|c|c|}
\hline & $\begin{array}{l}\text { Vertical ground } \\
\text { reaction force }\end{array}$ & Hip moment & Knee moment & Ankle moment \\
\hline PRS-20\% & $\begin{array}{l}6 \pm 2 \\
(11)\end{array}$ & $\begin{array}{c}12 \pm 4 \\
(22)\end{array}$ & $\begin{array}{c}11 \pm 4 \\
(19)\end{array}$ & $\begin{array}{l}7 \pm 3 \\
(14)\end{array}$ \\
\hline PRS & $\begin{array}{l}6 \pm 3 \\
(15)\end{array}$ & $\begin{array}{c}12 \pm 5 \\
(22)\end{array}$ & $\begin{array}{c}13 \pm 6 \\
(24)\end{array}$ & $\begin{array}{l}7 \pm 4 \\
(16)\end{array}$ \\
\hline PRS+20\% & $\begin{array}{l}6 \pm 2 \\
(14)\end{array}$ & $\begin{array}{c}11 \pm 3 \\
(17)\end{array}$ & $\begin{array}{c}10 \pm 5 \\
(27)\end{array}$ & $\begin{array}{l}8 \pm 4 \\
(18)\end{array}$ \\
\hline
\end{tabular}


